\title{
Low Concentrations of Nitric Oxide Increase Oxygen Affinity of Sickle Erythrocytes In Vitro and In Vivo
}

\author{
C. Alvin Head, , Carlo Brugnara," Ricardo Martinez-Ruiz, ${ }^{*}$ Robert M. Kacmarek, ${ }^{\star}$ Kenneth R. Bridges," David Kuter,, \\ Kenneth D. Bloch, ${ }^{\S}$ and Warren M. Zapol* \\ *Department of Anesthesia and Critical Care, ${ }^{\ddagger}$ Hematology and Oncology Unit, and ${ }^{\S}$ Cardiovascular Research Center of the Department \\ of Medicine, Massachusetts General Hospital, "Department of Pathology, Children's Hospital, "Department of Hematology, Brigham and \\ Women's Hospital, Harvard Medical School, Boston, Massachusetts 02115
}

\begin{abstract}
The hallmark of sickle cell disease (SCD) is the polymerization of deoxygenated sickle hemoglobin (HbS). In SCD patients, one strategy to reduce red blood cell (RBC) sickling is to increase $\mathrm{HbS}$ oxygen affinity. Our objective was to determine if low concentrations of nitric oxide (NO) gas would augment the oxygen affinity of RBCs containing homozygous $\mathrm{HbS}$ (SS). Blood containing normal adult hemoglobin (AA) or SS RBCs was incubated in vitro in the presence of varying concentrations of NO up to $80 \mathrm{ppm}$, and oxygen dissociation curves (ODCs) were measured. In addition, blood was obtained from three AA and nine SS volunteers, before and after breathing $80 \mathrm{ppm} \mathrm{NO}$ in air for $45 \mathrm{~min}$, and the ODCs were measured. Exposure of SS RBCs to $80 \mathrm{ppm}$ NO in vitro for $5 \mathrm{~min}$ or longer decreased the partial pressure of oxygen at which hemoglobin is $50 \%$ saturated with oxygen $\left(\mathrm{P}_{50}\right)$, an average of $15 \%(4.8 \pm 1.7 \mathrm{mmHg}$ mean $\pm \mathrm{SE} ; P<$ $0.001)$. The increase in SS RBC oxygen affinity correlated with the NO concentration. The $\mathrm{P}_{50}$ of AA RBCs was unchanged $(P>0.1)$ by $80 \mathrm{ppm}$ NO. In SS volunteers breathing $80 \mathrm{ppm}$ NO for $45 \mathrm{~min}$, the $P_{50}$ decreased $(P<0.001)$ by $4.6 \pm 2.0 \mathrm{mmHg}$. $60 \mathrm{~min}$ after NO breathing was discontinued, the $\mathrm{RBC} \mathrm{P}_{50}$ remained decreased in five of seven volunteers in whom the ODC was measured. There was no RBC $P_{50}$ change $(P>0.1)$ in AA volunteers breathing NO. Methemoglobin (Mhb) remained low in all subjects breathing NO (SS Mhb 1.4 $\pm 0.5 \%)$, and there was no correlation $(r=$ 0.02 ) between the reduction in $P_{50}$ and the change in Mhb. Thus, low concentrations of NO augment the oxygen affinity of sickle erythrocytes in vitro and in vivo without significant Mhb production. These results suggest that low concentrations of NO gas may offer an attractive new therapeutic model for the treatment of SCD. (J. Clin. Invest. 1997. 100: 1193-1198.) Key words: antisickling agents $\bullet \mathrm{P}_{50} \cdot$ therapy $\bullet$ anemia
\end{abstract}

Address correspondence to C. Alvin Head, M.D., Department of Anesthesia and Critical Care, Massachusetts General Hospital, Boston, MA 02114. Phone: 617-726-1880; FAX: 617-726-7536; E-mail: head@ etherdome.mgh.harvard.edu

Received for publication 4 February 1997 and accepted in revised form 10 June 1997.

J. Clin. Invest.

(C) The American Society for Clinical Investigation, Inc. 0021-9738/97/09/1193/06 \$2.00

Volume 100, Number 5, September 1997, 1193-1198

http://www.jci.org

\section{Introduction}

The primary features of sickle cell disease (SCD) ${ }^{1}$ include severe hemolytic anemia, frequent vasoocclusive episodes, and shortened longevity. SCD is caused by a single point mutation in the DNA encoding the sixth amino acid of the hemoglobin $\beta$ chain. The mutation results in the replacement of a negatively charged amino acid, glutamine, with a neutral hydrophobic residue, valine. Upon deoxygenation, sickle hemoglobin $(\mathrm{HbS})$ aggregates and produces a viscous gel composed of multistranded helical polymers, resulting in rigid and deformed red blood cells (RBCs). These RBCs have impaired ability to traverse the microcirculation, transiently or permanently blocking the microvasculature and decreasing oxygen supply to surrounding tissues. The resulting acute and chronic organ damage is a major cause of pain, morbidity, and mortality associated with SCD $(1,1 b)$.

Therapeutic strategies for SCD are based on reducing HbS polymerization by increasing the cellular concentration of hemoglobin $\mathrm{F}(\mathrm{HbF})$, reducing the cellular concentration of $\mathrm{HbS}$, or chemically modifying $\mathrm{HbS}$. As shown in the Multicenter Sickle Hydroxyurea study, the increase in $\mathrm{HbF}$ induced by hydroxyurea (HU) therapy was associated with a very significant reduction in pain rates, acute chest crises, and transfusion requirements (2). Oral clotrimazole has been shown to reduce sickle cell dehydration in a short-term study in patients with SCD (3). Combination therapy using agents with different mechanisms of action is being considered for the treatment of SCD.

Another therapeutic approach is based on reducing $\mathrm{HbS}$ polymerization by increasing the affinity of $\mathrm{HbS}$ for oxygen. As measured by the hemoglobin oxygen dissociation curve (ODC), homozygous HbS (SS) erythrocytes have markedly reduced affinity for oxygen as compared to normal adult hemoglobin (AA) erythrocytes containing hemoglobin A (HbA). The reduction in oxygen affinity of sickle erythrocytes is due to an increase in intraerythrocytic 2,3-diphosphoglycerate (DPG) concentrations as compared to normal HbA erythrocytes, and to the presence of $\mathrm{HbS}$ polymers (4-6). This decreased RBC oxygen affinity is reflected in an increase in the partial pressure of oxygen at which hemoglobin is half-saturated with oxygen $\left(\mathrm{P}_{50}\right)$. Compared to AA red cells, the tight $(\mathrm{T})$ deoxyhemoglobin conformational state is favored over the relaxed $(\mathrm{R})$

1. Abbreviations used in this paper: AA, normal adult hemoglobin; DPG, diphosphoglycerate; HbS, sickle hemoglobin; HU, hydroxyurea; Mhb, methemoglobin; NO, nitric oxide; ODC, oxygen dissociation curve; oxyHb, oxyhemoglobin; $\mathrm{P}_{50}$, partial pressure of oxygen at which hemoglobin is $50 \%$ saturated with oxygen; R, relaxed; RBC, red blood cell; $\mathrm{SCD}$, sickle cell disease; $\mathrm{SpO}_{2}$, pulse oximetry; $\mathrm{SS}$, homozygous sickle hemoglobin; $\mathrm{T}$, tight. 
oxyhemoglobin (oxyHb) conformation at any given oxygen tension in SS red cells. Polymerization only occurs when HbS is in the deoxyhemoglobin conformation. Sunshine et al. suggested that therapeutically significant inhibition of intracellular HbS polymerization could be accomplished by increasing the oxygen affinity (e.g., reducing $\mathrm{P}_{50}$ by $4 \mathrm{mmHg}$ ) (7).

Modification of hemoglobin affinity for oxygen has been shown to increase survival under hypoxic conditions in a transgenic mouse model of SCD (8). Other investigators have demonstrated that increasing oxygen affinity of $\mathrm{HbS}$ by exposure to sodium cyanate or carbon monoxide $(\mathrm{CO})$ reduces $\mathrm{HbS}$ RBC sickling in vitro $(9,10)$. However, these agents are too toxic for clinical use (11). High concentrations of NO have also been demonstrated to increase oxygen affinity in AA RBCs; however, significant methemoglobin (Mhb) was produced (12). The ability of low, nontoxic concentrations of NO gas to alter the oxygen affinity of SS erythrocytes has not been reported. In this study, the effect of low concentrations of NO on the oxygen affinity of erythrocytes with SS or AA was evaluated by measuring the ODC and $\mathrm{P}_{50}$ in vitro and in human subjects breathing low levels of NO. We report here that exposure of SS RBCs to low concentrations of $\mathrm{NO}$ gas in vitro and in vivo increases oxygen affinity without producing significant Mhb levels.

\section{Methods}

Subjects. All protocols were approved by the Massachusetts General Hospital Subcommittee on Human Studies, and all subjects gave signed informed consent. Three male AA volunteers (ages 25-40 yr) and nine (six males and three females) clinically stable SS volunteers (ages 18-36 yr) were studied. Three SS volunteers were receiving HU therapy and had been taking $\mathrm{HU}$ for $>6 \mathrm{mo}$.

$O D C$ determinations. $50 \mu \mathrm{l}$ whole blood was obtained by venipuncture from AA or SS volunteers and diluted with $4 \mathrm{ml}$ phosphate buffer, $10 \mu \mathrm{l}$ antifoam solution, and $20 \mu \mathrm{l} 20 \%$ albumin. The blood samples were desaturated by exposure to $100 \%$ nitrogen $\left(\mathrm{N}_{2}\right)$ gas and then reoxygenated with air using a Hemox analyzer (TCS Medical Products Co., Huntingdon Valley, PA) to measure the ODC, as reported previously (13). $\mathrm{P}_{50}$ was determined as the partial pressure of oxygen at $50 \%$ oxyHb saturation. To ensure the accuracy of repeated measures over time using SS RBCs, blood samples obtained from three SS volunteers had repeated ODC measurements made at 0,15 , 30 , and 60 min without $\mathrm{NO}$ gas exposure, and changes in $\mathrm{P}_{50}$ were not detected.

In vitro $N O$ exposure of $S S$ and $A A R B C s$. NO gas was added using a rotameter during $\mathrm{RBC}$ reoxygenation and displaced equal volumes of $\mathrm{N}_{2}$. Concentrations of $\mathrm{NO}$ were continuously monitored using an electrochemical analyzer (model SAAN TM-100; Taiyo Sanso, Tokyo, Japan), which was frequently calibrated by an NO chemiluminescence analyzer (model CLD-700 AL; ECO-Physics, Inc., Ann Arbor, MI). For all samples, ODCs were measured first using air to determine a baseline. RBCs were then exposed to air with 10,40 , or 80 ppm NO for 1-60 min, and ODCs were repeated. Mhb levels were measured before and after exposure to NO using a CO-Oximeter (model 270; Ciba Corning, Medfield, MA).

$N O$ inhalation in SS and AA volunteers. Three normal and nine SCD volunteers were studied. One SS volunteer was studied twice, with 1 mo between studies. Blood pressure, electrocardiogram, respiratory and heart rates, and pulse oximetry $\left(\mathrm{SpO}_{2}\right)$ were monitored continuously. Subjects breathed air and then $80 \mathrm{ppm}$ NO in air via a nonrebreathing circuit for $45 \mathrm{~min}$. Venous blood was sampled before and immediately after $\mathrm{NO}$ breathing. The normal subjects and seven SCD patients had an additional blood sample drawn $1 \mathrm{~h}$ after NO breathing. ODCs were measured ex vivo, as described above.
In addition, red cell ATP and 2,3-DPG concentrations were determined as reported previously (14). Mhb was measured using a COOximeter, as described above. Venous $\mathrm{pH}$ and blood gases were measured using a pH/blood gas analyzer (model 170; Ciba Corning). In two sickle cell volunteers (SS volunteers 9 and 10) and two HbA volunteers, we performed Mhb analysis by both the CO-Oximeter and spectrophotometrically, using a spectrophotometer (Cary 2000; Varian Corp., Sugarland, TX). This instrument did not show any production of Mhb at the absorption peak of $630 \mathrm{~nm}$ in blood of SS volunteers after NO therapy, even though the CO-Oximeter showed an increase in Mhb. Therefore, this commercial analyzer appears to overestimate the value of Mhb in NO-modified sickle erythrocytes.

Spectrophotometry. Whole blood samples taken before and after $\mathrm{NO}$ breathing were immediately frozen at $-80^{\circ} \mathrm{C}$ and transported on dry ice to Northeastern University (Dr. James Manning's laboratory), where they were stored at $-80^{\circ} \mathrm{C}$ until spectral analysis. Storage in this manner does not affect their integrity, since control studies showed that storage at this temperature for months does not cause formation of Mhb from oxyHb. Before analysis, the samples were thawed and centrifuged at 2,000 $\mathrm{g}$. The clear supernatant was then analyzed in two modes in a spectrophotometer (Cary 2000; Varian Corp.). This instrument is well-suited for this purpose because of its high quality optics and ability to measure absorbance values up to $4.0 \mathrm{U}$ with no deviation from linearity. In the first mode, the spectral bands at 577,560 , and $415 \mathrm{~nm}$ were measured, and their ratios to one another were calculated. For all samples (obtained from $\mathrm{HbA}$ and $\mathrm{HbS}$ volunteers before and after NO breathing), the spectra indicated only oxyHb. Nevertheless, this is an indirect manner of measuring Mhb, which has a separate absorbance band at $630 \mathrm{~nm}$, but an extinction coefficient only one-fifth that of oxyHb at $577 \mathrm{~nm}$, making its determination impossible from the first spectra. Therefore, it was necessary to use relatively concentrated hemoglobin samples (easily done in the Cary 2200) in which all other absorbance bands were off-scale, but any Mhb, if present, would be observed at $630 \mathrm{~nm}$. No Mhb was observed in any of the blood samples. An upper limit of $0.1 \%$ was estimated by determining what could have been observed at $630 \mathrm{~nm}$ (if present) with the off-scale values of the other wavelengths. Hence, using two criteria on the Cary 2200 (direct and indirect), no Mhb was measured in any of the blood samples.

Statistical analysis. Data are expressed as mean \pm SEM, except where indicated. Both paired and unpaired Student's $t$ test were used with a $P$ value $<0.05$ indicating statistical significance. All tests were two-sided. Correlations were evaluated by computing the Pearson correlation coefficient.

\section{Results}

In vitro NO exposure to $S S$ and $A A R B C s$. To determine the effect of low concentrations of NO gas on SS RBC oxygen affinity, blood from SS volunteers was incubated with varying concentrations of NO, and ODCs were measured. Exposure of SS RBCs to 80 ppm NO gas in air for 15 min increased oxygen affinity, producing a significant shift (towards normal) of the ODC (Fig. $1 A$ ). The shape of the ODC curve during NO exposure was maintained, suggesting that the cooperativity of oxygen binding was preserved. In RBCs containing SS, exposure to NO for 15 min decreased $(P<0.001)$ the $\mathrm{P}_{50}$ an average of $15 \%(4.8 \pm 1.7 \mathrm{mmHg}$; Fig. $1 B)$. The dose of NO administered was directly proportional to the increase in SS RBC oxygen affinity (Fig. $1 C$ ). The effect of $\mathrm{NO}$ exposure on $\mathrm{P}_{50}$ was dependent upon the duration of exposure, with 5 min producing a significant reduction (Fig. $1 D$ ). When SS RBCs were exposed to $80 \mathrm{ppm} \mathrm{NO}$ for $15 \mathrm{~min}$ and then exposed to air without $\mathrm{NO}$, the reduction in $\mathrm{P}_{50}$ persisted for at least $2 \mathrm{~h}$ (data not shown). Exposure to $80 \mathrm{ppm}$ NO for $15 \mathrm{~min}$ did not alter the oxygen affinity of normal RBCs containing AA (Fig. $1 B$ ). In 


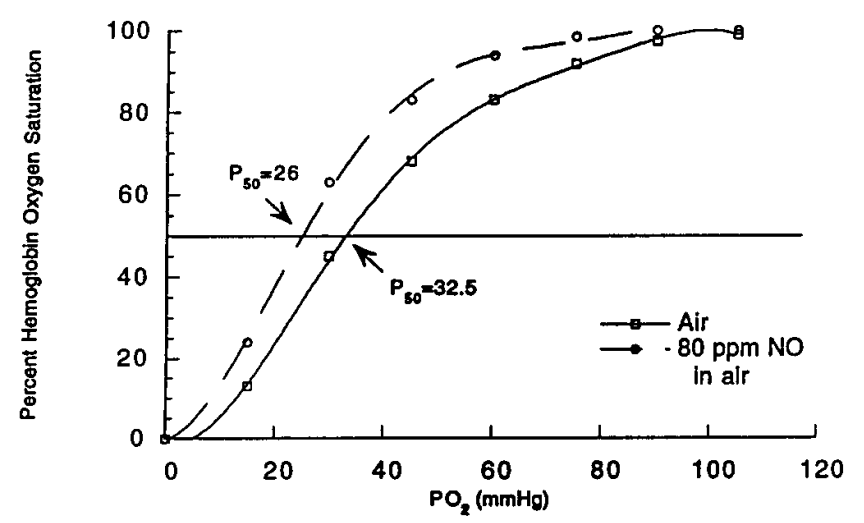

B

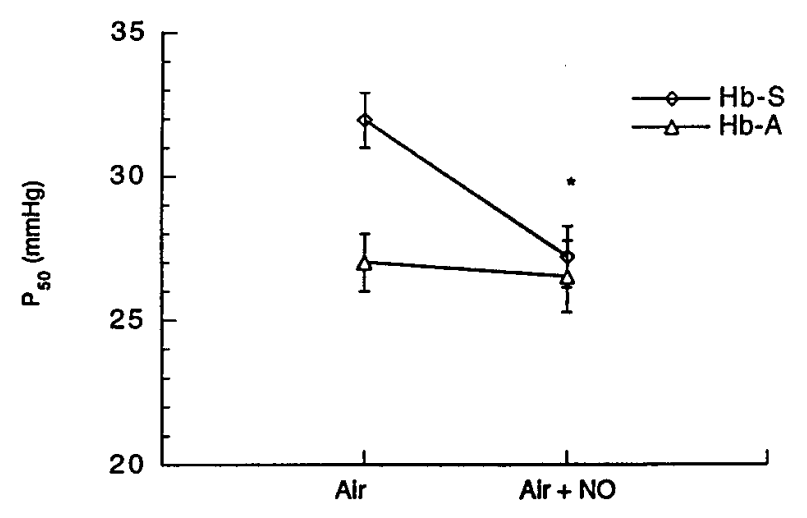

C

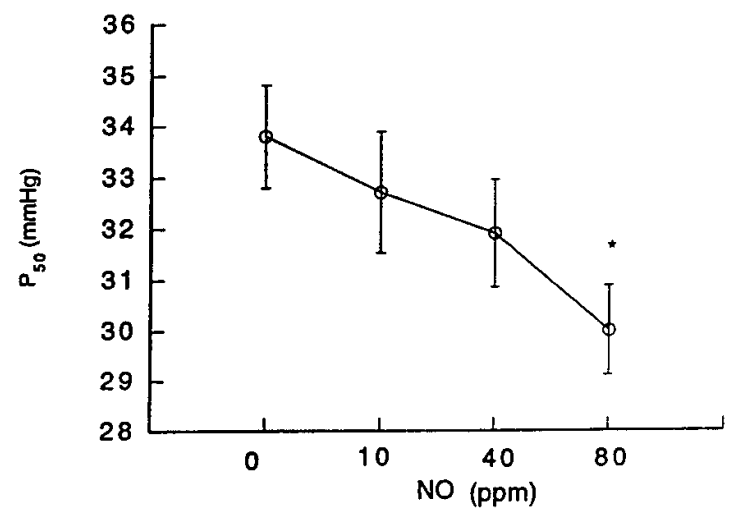

$\mathbf{D}$

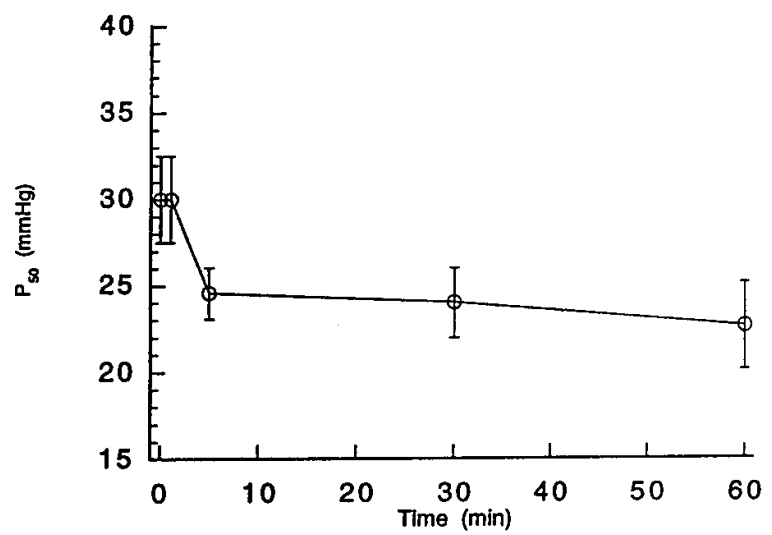

Figure 1. In vitro exposure to low concentrations of NO gas increased SS RBC oxygen affinity. ( $A$ ) Representative SS RBC ODC without ( $\square$ ) and with $(O) 15 \mathrm{~min}$ of exposure to $80 \mathrm{ppm}$ NO in air. Exposure to NO shifted the SS RBC ODC to the left, and the sigmoid shape was maintained. $(B)$ The mean value $( \pm \mathrm{SE}) \mathrm{RBC} \mathrm{P}_{50}$ of blood samples $\mathrm{SS}(\diamond, n=10)$ and AA $(\triangle, n=3)$ were measured after exposure to air or air with $80 \mathrm{ppm}$ NO for $15 \mathrm{~min}$. NO exposure decreased the SS RBC $\mathrm{P}_{50}$ by $15 \%$ or $5 \mathrm{mmHg}(* P<0.001)$, as compared to the ODC derived without NO exposure. There was no difference $(P>0.05)$ in the AA RBC $P_{50}$ with or without NO. $(C)$ The SS RBC $\mathrm{P}_{50}( \pm \mathrm{SE})$ of SS volunteers $(n=3)$ exposed to air or air containing concentrations of 10,40 , or $80 \mathrm{ppm} \mathrm{NO}$ for $15 \mathrm{~min}$. There was a reduction of SS RBC $\mathrm{P}_{50}$ at each dose; however, only the $80 \mathrm{ppm}$ NO dose was significant $(* P<0.05)$. $(D)$ The mean $\mathrm{RBC}_{50}$ using blood from SS volunteers $(n=3)$ exposed to air or air with $80 \mathrm{ppm} \mathrm{NO}$ added for periods of $1,5,30$, and $60 \mathrm{~min}$. No change in $\mathrm{P}_{50}$ was noted after $1 \mathrm{~min}$. However, a reduction in the $\mathrm{SS} R B C \mathrm{P}_{50}$ was noted after $5 \mathrm{~min}$, and this was unchanged after longer exposures to NO. RBC Mhb levels measured with the Corning CO-Oximeter were low $(<3 \%)$ after $60 \mathrm{~min}$ of $80 \mathrm{ppm}$ NO exposure.

these experiments, exposure of RBCs containing SS or AA to $80 \mathrm{ppm}$ NO for up to $60 \mathrm{~min}$ produced low Mhb levels $(<3 \%$ by CO-Oximeter). These results suggest that in vitro, the oxygen affinity of SS erythrocytes is uniquely sensitive to low concentrations of NO.

$N O$ inhalation in SS and AA volunteers. To determine whether low concentrations of NO could alter $\mathrm{HbS}$ in vivo, blood $\mathrm{P}_{50}$ from AA and SS volunteers was measured before and after breathing $80 \mathrm{ppm} \mathrm{NO}$ in air for $45 \mathrm{~min}$. In SS volunteers breathing 80 ppm NO, the RBC $\mathrm{P}_{50}$ was decreased $(P<$ $0.001)$, with an average reduction in $\mathrm{RBC}_{50}$ of $4.6 \pm 2 \mathrm{mmHg}$ (Fig. 2 and Table I). In contrast, the $\mathrm{RBC}_{50}$ did not change ( $\leq 1 \mathrm{mmHg} ; P=\mathrm{NS}$ ) in the AA volunteers breathing NO. One SS volunteer was studied twice with 1 mo between studies and demonstrated a significant reduction in $\mathrm{P}_{50}$ on both occasions. In seven SS volunteers, the ODC was measured $1 \mathrm{~h}$ after
NO inhalation was discontinued. In five of the seven, the RBC $\mathrm{P}_{50}$ remained decreased, suggesting that the effect of $\mathrm{NO}$ on the oxygen affinity of SS RBCs may persist after NO is discontinued (Fig. 2 and Table I).

In all subjects breathing $80 \mathrm{ppm} \mathrm{NO}$ in air for $45 \mathrm{~min}$, the RBC ATP and 2,3-DPG concentrations did not change. The blood pressure, respiratory and heart rates, $\mathrm{SpO}_{2}$, venous blood $\mathrm{pH}$, and electrocardiogram were unchanged during NO breathing. Volunteers with SS red cells had a higher baseline Mhb $(0.5 \pm 0.2 \%)$ compared to those with AA red cells $(0.1 \pm 0.1 \%)$. Exposure to $\mathrm{NO}$ led to a small but significant increase in Mhb levels in both SS $(1.4 \pm 0.7 \%)$ and AA $(0.7 \pm 0.1 \%)$ volunteers, with a return toward baseline values at 60 min after NO exposure $(0.6 \pm 0.3$ and $0.2 \pm 0.1 \%$ for SS and AA volunteers, respectively). There was no correlation between the increase in Mhb levels and the decrease in $\mathrm{P}_{50}$ values 


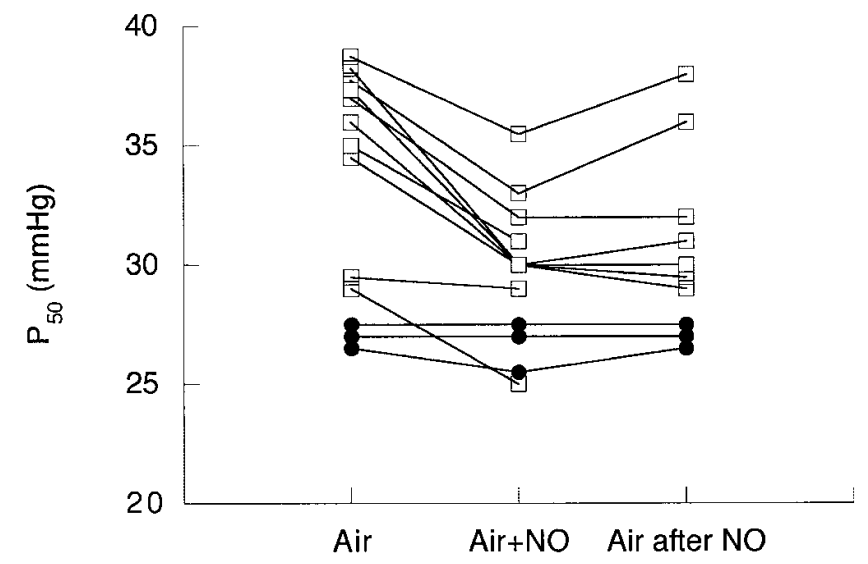

Figure 2. Inhaled NO increased RBC oxygen affinity in SS volunteers. The average reduction of the RBC $\mathrm{P}_{50}$ of 10 studies with nine stable SS volunteers $(\square)$ was $\sim 5 \mathrm{mmHg}$ (range 3-8 $\mathrm{mmHg} ; P<$ 0.001 ) after $80 \mathrm{ppm} \mathrm{NO}$ breathing for $45 \mathrm{~min}$. In one SS volunteer, the $\mathrm{RBC}_{50}$ did not change. Normal volunteers $(\bullet)$ had no change $(\leq 1 \mathrm{mmHg})$ in the AA RBC $\mathrm{P}_{50}$ during NO breathing. Blood samples were taken from three AA and seven SS volunteers while breathing air $1 \mathrm{~h}$ after NO breathing. Five of seven SS volunteers maintained the $\mathrm{RBC} \mathrm{P}_{50}$ reduction for at least $1 \mathrm{~h}$. In all subjects, erythrocytic 2,3-DPG and ATP levels, venous $\mathrm{pH}$, and blood gas tensions did not change after $45 \mathrm{~min}$ of NO breathing. In all subjects, there were no clinical side effects noted. The mean Mhb levels after 45 min of NO breathing were low (see Table I) for SS RBCs and returned to baseline after $60 \mathrm{~min}$, though NO effects persisted.
(Fig. 3, $n=10, r=0.02$ ). In fact, the shift in $\mathrm{P}_{50}$ persisted at 60 min after NO exposure in five of seven SS volunteers tested, while the Mhb levels had returned to baseline values. In addition, in SS volunteers 9 and 10, Mhb levels were measured with spectrophotometric analysis. These two patients had among the highest changes in Mhb levels after NO treatment. After 45 min of NO treatment, there was no Mhb detectable at $630 \mathrm{~nm}$.

\section{Discussion}

The most important finding of this study is that hemoglobin oxygen affinity increases when SS erythrocytes are exposed to low concentrations of NO. This effect was observed when RBCs were exposed to NO in vitro or during NO inhalation in vivo. Exposure to $\mathrm{NO}$ did not produce clinically significant Mhb levels. Increased SS RBC oxygen affinity was observed within 5 min of $\mathrm{NO}$ exposure in vitro, and the increase persisted for $2 \mathrm{~h}$. In five of seven SS volunteers in whom it was measured, the $\mathrm{RBC} \mathrm{P}_{50}$ remained decreased at least $60 \mathrm{~min}$ after NO breathing was discontinued. In contrast, AA RBC oxygen affinity was unaffected by exposure to low concentrations of NO, either in vitro or in vivo.

The mechanisms by which low concentrations of NO augment the oxygen affinity of SS erythrocytes but not AA erythrocytes are unknown. The reaction between $\mathrm{NO}$ and the heme moiety of hemoglobin has been studied in great detail using extremely high NO concentrations (up to $100 \%)(12,14,15)$. However, there is no information available on the effects of

Table I. Effects of Inhaled NO in SS and AA Volunteers

\begin{tabular}{|c|c|c|c|c|c|c|c|c|c|c|c|c|c|}
\hline \multirow[b]{2}{*}{ Trial } & \multirow[b]{2}{*}{ Age } & \multirow[b]{2}{*}{ Sex } & \multirow[b]{2}{*}{ Transfusion } & \multirow[b]{2}{*}{$\mathrm{HU}$} & \multirow{2}{*}{$\begin{array}{l}\text { Crisis } \\
\text { per yr }\end{array}$} & \multirow{2}{*}{$\begin{array}{c}\mathrm{Hb} \\
(\mathrm{g} / \mathrm{dl})\end{array}$} & \multirow[b]{2}{*}{$\mathrm{SpO}_{2}$} & \multicolumn{3}{|c|}{$\mathrm{P}_{50}(\mathrm{mmHg})$} & \multicolumn{3}{|c|}{ Mhb\% } \\
\hline & & & & & & & & Baseline & Inhaled NO & Post NO & Baseline & Inhaled NO & Post NO \\
\hline \multicolumn{14}{|c|}{ SS Volunteers } \\
\hline 1 & 27 & M & Yes & No & 1 & 6.5 & $97 \%$ & 38.5 & 35.5 & 38 & 0.5 & 1.1 & 0.7 \\
\hline 2 & 32 & $\mathrm{M}$ & No & No & 2 & 6.9 & $96 \%$ & 37 & 32 & 32 & 0.3 & 2.7 & 0.4 \\
\hline 3 & 33 & M & No & No & 1 & 10.1 & $97 \%$ & 35 & 30 & 30 & 0.8 & 1.3 & 1.3 \\
\hline 4 & 30 & $\mathrm{M}$ & Yes & No & 8 & 8.2 & $99 \%$ & 36.5 & 30 & 29 & 0.3 & 1.1 & 0.5 \\
\hline 5 & 27 & $\mathrm{~F}$ & No & Yes & 12 & 6 & $94 \%$ & 38 & 30 & 31 & 0.3 & 1.2 & 0.1 \\
\hline 6 & 36 & M & No & No & 3 & 6.1 & $93 \%$ & 29 & 29 & $\mathrm{x}$ & 0.6 & 1.2 & 0.8 \\
\hline 7 & 30 & M & Yes & No & 14 & 6.1 & $90 \%$ & 29 & 25 & $\mathrm{x}$ & 0.5 & 1.1 & 0.6 \\
\hline 8 & 18 & $\mathrm{~F}$ & No & Yes & 10 & 6.9 & $97 \%$ & 36.5 & 33 & 36 & 0.3 & 1.5 & 0.7 \\
\hline 9 & 28 & $\mathrm{~F}$ & No & Yes & 9 & 6 & $98 \%$ & 37 & 30 & 30 & 0.9 & 1.5 & 0.5 \\
\hline$* 10$ & 27 & $\mathrm{~F}$ & Yes & Yes & 12 & 7.3 & $95 \%$ & 35 & 31 & $\mathrm{x}$ & 0.9 & 3.2 & 0.5 \\
\hline \multicolumn{14}{|c|}{ AA Volunteers } \\
\hline 1 & 25 & $\mathrm{M}$ & NA & NA & NA & 13.2 & 100 & 27 & 27 & 27 & 0.1 & 0.8 & 0.2 \\
\hline 2 & 40 & M & NA & NA & NA & 12.5 & 100 & 26.5 & 25.5 & 26.5 & 0.1 & 0.8 & 0.3 \\
\hline 3 & 37 & $\mathrm{M}$ & NA & NA & NA & 13 & 100 & 27 & 27 & 27 & 0.2 & 0.5 & 0.2 \\
\hline
\end{tabular}

Nine SS volunteers were studied (*SS volunteer data 5 and 10 are from the same patient with 1 mo between studies and after a blood transfusion). Transfusion is yes if blood was received within 1 mo of study date. HU was not an exclusion to our study. SpO $\mathrm{O}_{2}$ was monitored continuously during the study. Crisis over the past 12 mo is shown as well as total hemoglobin $(H b)$ level at time of study. The average reduction of the RBC $\mathrm{P}_{50}$ of 10 studies with nine stable $\mathrm{SS}$ volunteers was $\sim 5 \mathrm{mmHg}$ (range $3-8 \mathrm{mmHg} ; P<0.001$ ) after 80 ppm NO breathing fo $45 \mathrm{~min}$. In one $\mathrm{SS}$ volunteer, the $\mathrm{RBC} \mathrm{P}_{50}$ did not change. Normal volunteers had no change $(\leq 1 \mathrm{mmHg})$ in the AA RBC $\mathrm{P}_{50}$ during NO breathing. Blood samples were taken from three AA and seven SS volunteers while breathing air $1 \mathrm{~h}$ after $\mathrm{NO}$ breathing had been discontinued. Five of seven SS volunteers maintained the RBC $\mathrm{P}_{50}$ reduction for at least $1 \mathrm{~h}$. In all subjects, erythrocytic 2,3-DPG and ATP levels, venous pH, and blood gas tensions did not change after 45 min of NO breathing. In all subjects, there were no clinical side effects noted. The mean Mhb levels (CO-Oximeter) after 45 min of NO breathing were low $(1.4 \pm 0.5 \%)$ for SS RBCs and returned to baseline after $60 \mathrm{~min}$, though NO effects persisted in five of seven patients evaluated. 


\section{Changes from baseline: MHb vs. P50}

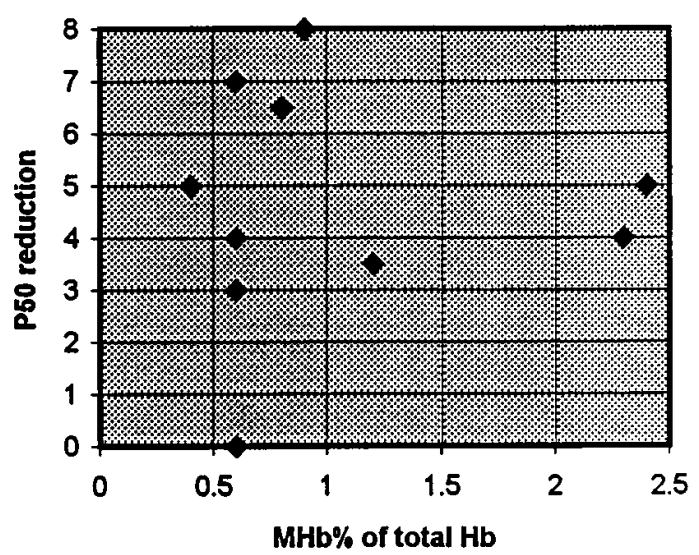

Figure 3. Change in $\mathrm{P}_{50}$ versus change in Mhb levels (CO-Oximeter) induced by inhaled NO in SS volunteers. Change in $\mathrm{P}_{50}$ was calculated from the difference between the value obtained after $45 \mathrm{~min}$ of breathing $80 \mathrm{ppm}$ of $\mathrm{NO}$ in air and the baseline value for each of the $\mathrm{SS}$ volunteers. Mhb levels were measured with the $\mathrm{CO}$-Oximeter after $45 \mathrm{~min}$ of breathing $80 \mathrm{ppm}$ of $\mathrm{NO}$ in air, and expressed as percentage of the total $\mathrm{Hb}$. No correlation could be established between these two parameters, $\mathrm{MHb}$ and $\mathrm{P}_{50}(r=0.024)$.

low doses of NO $(80 \mathrm{ppm}=0.008 \%)$ on sickle erythrocytes. Breathing low concentrations of NO has been used safely to dilate the pulmonary vasculature in various pulmonary disorders, such as adult respiratory distress syndrome and pulmonary hypertension $(16,17)$. NO binds avidly to the heme moiety in hemoglobin and releases more slowly than oxygen (18, 19). Therefore, NO may increase the tendency for the $\mathrm{HbS}$ conformation to be maintained in the $\mathrm{R}$ (high affinity) state. Since only deoxyhemoglobin $\mathrm{S}$ (i.e., T state $\mathrm{HbS}$ ) polymerizes, any experimental or therapeutic intervention that favors the $\mathrm{R}$ over the $\mathrm{T}$ state may result in a reduction in $\mathrm{HbS}$ polymerization. NO modification of $\mathrm{HbS}$ may reduce polymerization and thereby explain the increase of oxygen affinity in SS red cells while having no effect on oxygen affinity of AA red cells. By comparison, potassium cyanate modifies the oxygen affinity of both SS and AA red cells to the same extent in vitro (9). Therefore, the selective effect of NO on SS RBCs indicates a different mechanism (e.g., as a polymerization inhibitor for $\mathrm{HbS}$, see Fig. $1 B$ ), than an oxygen-based HbS modifier like potassium cyanate.

Another possibility is that $\mathrm{NO}$ increases erythrocyte $\mathrm{Mhb}$ levels, thereby altering oxygen affinity (20-22). However, since our in vitro and in vivo studies recorded only very low levels of Mhb in both AA and SS RBCs, it is unlikely that Mhb contributed to the selective increase in HbS RBC oxygen affinity. Moreover, we found no correlation between Mhb and $\mathrm{P}_{50}$ changes (Fig. 3), and the decrease in $\mathrm{P}_{50}$ persisted $60 \mathrm{~min}$ after exposure to NO, while the Mhb levels had returned to baseline values. Another theoretical concern would be the generation of additional hemoglobin oxidation products during NO breathing. Detailed spectrophotometric analysis from two SS volunteers after NO breathing failed to show any increase in Mhb or the production of abnormal hemoglobin or any additional hemoglobin oxidation products. However, additional studies are needed to evaluate this issue carefully.
NO can also affect other portions of the hemoglobin molecule by forming an adduct. Moriguchi et al. showed that the amino-terminal valine and possibly other amino groups of $\mathrm{HbA}$ within the 2,3-DPG cleft are modified by NO exposure, becoming more electronegative (23). HbS carries a Glu $\rightarrow \mathrm{Val}$ substitution at $\beta 6$, making $\mathrm{HbS}$ less negative than $\mathrm{HbA}$. Since this site determines HbS polymerization, NO-mediated modification might reduce intracellular polymerization, thereby increasing $\mathrm{HbS}$ oxygen affinity.

Thiol groups (such as $\beta 93$ Cys) also interact with NO, producing S-nitrosothiols which may play a role in the control of vascular tone (24). Interestingly, Hb Okazaki (a $\beta 93$ Cys-Arg variant) demonstrates increased oxygen affinity (25). It is possible that the oxygen affinity of S-nitrosothiol $\mathrm{HbS}$ behaves like that of $\mathrm{Hb}$ Okazaki. Moreover, $\beta 93$ Cys plays a crucial role in both hemoglobin oxygen affinity and $\mathrm{HbS}$ polymerization: when this residue is reacted with a thiol reagent, a significant reduction in both $\mathrm{P}_{50}$ and $\mathrm{HbS}$ polymer formation is observed $(26,27)$.

We found no increase in oxygen affinity of AA RBCs after exposure to $80 \mathrm{ppm} \mathrm{NO}$ gas. However, Kon et al. have shown that high concentrations of $\mathrm{NO}$ can increase oxygen affinity in HbA RBCs (12). Of note, Briehl and Salhany (18) observed that high concentrations $(100 \%)$ of $\mathrm{NO}$ in the presence of inositol hexaphosphate promoted the gelation of $\mathrm{HbS}$ in vitro, and suggested that $\mathrm{NO}$ induces the switch of $\mathrm{HbS}$ from the R to the T conformational state. Our data show that low concentrations of $\mathrm{NO}(80 \mathrm{ppm}=0.008 \% \mathrm{NO})$ do not promote an $\mathrm{R}$ to $\mathrm{T}$ switch. This apparent discrepancy may be related to the very low concentrations of $\mathrm{NO}$ that we studied.

To assess the ability of NO to increase $\mathrm{HbS}$ oxygen affinity in vivo, we measured the ODCs in blood from SS volunteers before and after NO breathing. We chose this approach for the administration of NO because RBCs are efficiently exposed to NO gas as they transit the lungs, and because inhaled NO does not produce systemic vasodilation $(16,28,29)$. Our in vitro analysis suggested $\mathrm{NO}$ gas could be safely administered to SCD patients without detrimental effects on SS RBCs. In contrast, NO donor compounds would be expected to decrease systemic perfusion pressure and could aggravate the occlusive phenomena associated with SCD. In this study, nine SS volunteers breathed $80 \mathrm{ppm} \mathrm{NO}$ for $45 \mathrm{~min}$ without significant methemoglobinemia, systemic hypotension, or any other adverse effects. Moreover, measurements of ODCs ex vivo suggested that NO inhalation rapidly and markedly increases SS RBC oxygen affinity, an effect which persisted for at least $1 \mathrm{~h}$ after NO breathing in five of seven SS volunteers studied. However, a theoretical concern of NO therapy involves nonuniform modification of $\mathrm{HbS}$ within the RBC, where unmodified HbS may unload more oxygen, suggesting that reaching a critical concentration of intracellular NO-modified HbS could be important. No study yet has shown that nonuniform HbS modification has enhanced RBC sickling either in vitro or in vivo. Another concern for NO therapy is the possibility of a reduction in oxygen carrying capacity. However, this is unlikely to be clinically significant, since we did not observe a reduction in oxygen saturation during NO breathing, and neither did two large clinical trials using NO therapy in hypoxic patients $(30,31)$.

Another theoretical concern for the increase in oxygen affinity with $\mathrm{NO}$ would be an increase in hematocrit or blood viscosity. This could partially negate the benefit of the increased oxygen affinity. However, there are no prior studies 
for comparison. We did not evaluate the oxygen affinity beyond $1 \mathrm{~h}$ after NO breathing had stopped. Therefore, we do not know the duration of NO's effect on HbS RBCs beyond $1 \mathrm{~h}$ in vivo. Additional studies are needed in the mouse model and in patients to determine effectively the duration of NO's effect and its long-term outcome.

In summary, our results demonstrate that inhaling low concentrations of NO gas increases the oxygen affinity of SS $\mathrm{RBCs}$ in vitro and in vivo. Low concentrations of NO did not alter oxygen affinity of AA RBCs, suggesting the effect of NO was selective for SS RBCs. Similar effects of NO on SS RBC oxygen affinity were observed in SS volunteers breathing NO gas, and were not associated with significant Mhb levels, changes in RBC 2,3-DPG, ATP, or systemic hypotension. Increases in oxygen affinity were also found in some of our SS volunteers receiving $\mathrm{HU}$ therapy, suggesting combination therapy is possible. Our studies were conducted over short time periods, and additional studies are needed to determine the long-term effects of NO therapy in patients with SCD. However, because interventions designed to increase SS erythrocyte oxygen affinity decrease RBC sickling, our results suggest that breathing low concentrations of NO gas may represent a novel therapeutic approach to the treatment of SCD.

\section{Acknowledgments}

The authors thank Stanley J. Nyarko, Natasha Mangny, Eric Roux, and Dr. Garland Cowan for data collection, and Professors Steven Tannenbaum, Paul Skipper, and Pete Wishnok at the Massachusetts Institute of Technology and Dr. H. Franklin Bunn of Harvard Medical School for helpful discussions. We thank Dr. James Manning of Northeastern University for performing spectrophotometry and for helpful comments.

This work was supported by US Public Health Service grants HL42397, HL-55377, and HL-15157. Dr. K.D. Bloch is an Established Investigator of the American Heart Association.

\section{References}

1. Eaton, W.A., and J. Hofrichter. 1987. Hemoglobin S gelation and sickle cell disease. Blood. 70:1245-1266.

1b. Noguchi, C.T., G.P. Rodgers, and A.N. Schechter. 1989. Intracellular polymerization. Disease severity and therapeutic predictions. Ann. NY Acad. Sci. 565:75-82.

2. Charache, S., M.L. Terrin, R.D. Moore, G.J. Dover, F.B. Barton, S.V. Eckert, R.P. McMahon, and D.R.Bonds. 1995. Effect of hydroxyurea on the frequency of painful crises in sickle cell anemia. N. Engl. J. Med. 332:1317-1322.

3. Brugnara, C., B. Gee, C. Armsby, S. Kurth, M. Sakamoto, N. Rifai, S.L. Alper, and O.S. Platt. 1996. Therapy with oral clotrimazole induces inhibition of the Gardos channel and reduction of erythrocyte dehydration in patients with sickle cell disease. J. Clin. Invest. 97:1227-1234.

4. Eaton, W.A., and J. Hofrichter. 1990. Sickle cell hemoglobin polymerization. Adv. Protein Chem. 40:63-79.

5. Poillon, W.N., B.C. Kim, R.J. Labotka, C.U. Hicks, and J.A. Kark. 1995. Antisickling effects of 2,3-diphosphoglycerate depletion. Blood. 85:3289-3296.

6. Benesch, R.E., R. Edalji, S. Kwong, and R. Benesch. 1978. Oxygen affinity as an index of hemoglobin S polymerization: a new micromethod. Anal. Bio- chem. 89:162-173

7. Sunshine, H.R., J. Hofrichter, and W.A. Eaton. 1978. Requirements for therapeutic inhibition of sickle haemoglobin gelation. Nature (Lond.). 275: 238-240.

8. Trudel, M., M.E. De Paepe, N. Chretien, N. Sadane, J. Jacmain, M. Sorette, T. Hoang, and Y. Beuzard. 1994. Sickle cell disease of transgenic SAD mice. Blood. 84:3189-3197.

9. De Furia, F.G., D.R. Miller, A. Cerami, and J.M. Manning. 1972. The effects of cyanate in vitro on red blood cell metabolism and function in sickle cell anemia. J. Clin. Invest. 51:566-574.

10. Butler, E. 1975. The effect of carbon monoxide on red cell life span in sickle cell disease. Blood. 46:253-255.

11. Bunn, H.F., and B.G. Forget. editors. 1986. Hemoglobin: Molecular Genetics and Clinical Aspects. W.B. Saunders Company, Philadelphia.

12. Kon, K., N. Maeda, and T. Shiga. 1977. Effect of nitric oxide on the oxygen transport of human erythrocytes. J. Toxicol. Environ. Health. 2:1109-1113.

13. Guarnone, R., E. Centenara, and G. Barosi. 1995. Performance characteristics of Hemox-Analyzer for assessment of the hemoglobin dissociation curve. Haematologica. 80:426-430.

14. Poillon, W.N., M.D. Robinson, and B.C. Kim. 1985. Deoxygenated sickle hemoglobin: modulation of its solubility by 2,3-diphosphoglycerate and other allosteric polyanions. J. Biol. Chem. 260:13897-13900.

15. Moore, E.G., and Q.H. Gibson. 1976. Cooperativity in the dissociation of nitric oxide from hemoglobin. J. Biol. Chem. 251:2788-2794.

16. Rossaint, R., J. Falke, F. Lopez, K. Slama, U. Pison, and W. Zapol. 1993. Inhaled nitric oxide for the adult respiratory distress syndrome. N. Engl. J. Med. 328:399-405.

17. Pepke-Zaba, J., T.W. Higenbottam, A.T. Dinu-Xuan, D. Stone, and J. Wallwork. 1991. Inhaled nitric oxide as a cause of selective pulmonary vasodilation in pulmonary hypertension. Lancet. 338:1173-1174.

18. Briehl, R.W., and J.M. Salhany. 1975. Gelation of sickle hemoglobin. III. Nitrosyl hemoglobin. J. Mol. Biol. 96:733-743.

19. Rimar, S., and C.N. Gillis. 1993. Selective pulmonary vasodilation by inhaled nitric oxide is due to hemoglobin inactivation. Circulation. 87:81-87.

20. Beutler, E. 1961. The effect of methemoglobin formation in sickle cell disease. J. Clin. Invest. 40:1856-1871.

21. Sharma, V.S., T.G. Traylor, and R. Gardiner. 1987. Reaction of nitric oxide with heme proteins and model compounds of hemoglobin. Biochemistry. 26:3837-3843.

22. Briehl, R.W., and S.M. Ewert. 1974. Gelation of sickle cell haemoglobin. II. Methaemoglobin. J. Mol. Biol. 89:759-766.

23. Moriguchi, M., L.R. Manning, and J.M. Manning. 1992. Nitric oxide can modify amino acid residues in proteins. Biochem. Biophys. Res. Commun. 183: 598-604.

24. Jia, L., C. Bonaventura, J. Bonaventura, and J.S. Stamler. 1996. S-nitrosohaemoglobin: a dynamic activity of blood involved in vascular control. Nature (Lond.). 380:221-226.

25. Harano, K., T. Harano, S. Shibata, S. Ueda, H. Mori, and M. Seki. 1984. $\mathrm{Hb}$ Okazaki [beta-93(F8) Cys-Arg], a new hemoglobin variant with increased oxygen affinity and instability. FEBS (Fed. Eur. Biochem. Soc.) Lett. 173:45-47.

26. Garel, M.C., C. Domenget, J. Caburi-Martin, C. Prehu, F. Galacteros, and Y. Beuzard. 1986. Covalent binding of glutathione to hemoglobin. I. Inhibition of hemoglobin S polymerization. J. Biol. Chem. 261:14704-14709.

27. Craescu, C.T., C. Poyart, C. Schaeffer, M.C. Garel, J. Kister, and Y. Beuzard. 1986. Covalent binding of glutathione to hemoglobin. II. Functional consequences and structural changes reflected in NMR spectra. J. Biol. Chem. 261:14710-14716.

28. Frostell, C., M.D. Fratacci, J.C. Wain, R. Jones, and W.M. Zapol. 1991 Inhaled nitric oxide. A selective pulmonary vasodilator reversing hypoxic pulmonary vasoconstriction. Circulation. 83:2038-2047.

29. Zapol, W.M., S. Rimar, N. Gillis, M. Marletta, and C.H. Bosken. 1994 Nitric oxide and the lung. Am. J. Respir. Crit. Care Med. 149:1375-1380.

30. The Neonatal Inhaled Nitric Oxide Study Group. 1997. Inhaled nitric oxide in full-term and nearly full-term infants with hypoxic respiratory failure. N. Engl. J. Med. 336:597-604.

31. Roberts, J.D., J.R. Fineman, F.C. Morin III, P.W. Shaul, S. Rimar, M.D. Schreiber, R.A. Polin, M.S. Zwass, M.M. Zayek, I. Gross, et al. 1997. Inhaled nitric oxide and persistent pulmonary hypertension of the newborn. $N$. Engl. J. Med. 336:605-610. 